\title{
Analytical Calculation of Stored Electrostatic Energy per Unit Length for an Infinite Charged Line and an Infinitely Long Cylinder in the Framework of Born-Infeld Electrostatics
}

\author{
S. K. Moayedi, M. Shafabakhsh, and F. Fathi \\ Department of Physics, Faculty of Sciences, Arak University, Arak 38156-8-8349, Iran \\ Correspondence should be addressed to S. K. Moayedi; s-moayedi@araku.ac.ir
}

Received 28 December 2014; Accepted 7 March 2015

Academic Editor: George Siopsis

Copyright ( $\odot 2015$ S. K. Moayedi et al. This is an open access article distributed under the Creative Commons Attribution License, which permits unrestricted use, distribution, and reproduction in any medium, provided the original work is properly cited. The publication of this article was funded by SCOAP $^{3}$.

\begin{abstract}
More than 80 years ago, Born-Infeld electrodynamics was proposed in order to remove the point charge singularity in Maxwell electrodynamics. In this work, after a brief introduction to Lagrangian formulation of Abelian Born-Infeld model in the presence of an external source, we obtain the explicit forms of Gauss's law and the energy density of an electrostatic field for Born-Infeld electrostatics. The electric field and the stored electrostatic energy per unit length for an infinite charged line and an infinitely long cylinder in Born-Infeld electrostatics are calculated. Numerical estimations in this paper show that the nonlinear corrections to Maxwell electrodynamics are considerable only for strong electric fields. We present an action functional for Abelian Born-Infeld model with an auxiliary scalar field in the presence of an external source. This action functional is a generalization of the action functional which was presented by Tseytlin in his studies on low energy dynamics of D-branes (Nucl. Phys. B469, 51 (1996); Int. J. Mod. Phys. A 19, 3427 (2004)). Finally, we derive the symmetric energy-momentum tensor for Abelian Born-Infeld model with an auxiliary scalar field.
\end{abstract}

\section{Introduction}

Maxwell electrodynamics is a very successful theory which describes a wide range of macroscopic phenomena in electricity and magnetism. On the other hand, in Maxwell electrodynamics, the electric field of a point charge $q$ at the position of the point charge is singular; that is,

$$
\mathbf{E}(\mathbf{x})=\frac{q}{4 \pi \epsilon_{0}|\mathbf{x}|^{2}} \frac{\mathbf{x}}{|\mathbf{x}|} \stackrel{|\mathbf{x}| \rightarrow 0}{\longrightarrow} \infty .
$$

Also, in Maxwell electrodynamics, the classical self-energy of a point charge is

$$
U=\frac{q^{2}}{8 \pi \epsilon_{0}} \int_{0}^{\infty} \frac{d r}{r^{2}} \longrightarrow \infty .
$$

More than 80 years ago, Born and Infeld proposed a nonlinear generalization of Maxwell electrodynamics [1]. In their generalization, the classical self-energy of a point charge was a finite value [1-6]. Recent studies in string theory show that the dynamics of electromagnetic fields on $D$-branes can be described by Born-Infeld theory [7-10]. In a paper on BornInfeld theory [8], the concept of a BIon was introduced by Gibbons. BIon is a finite energy solution of a nonlinear theory with a distributional source. Today, many physicists believe that the dark energy in our universe can be described by a Born-Infeld type scalar field [11]. The authors of [12] have presented a non-Abelian generalization of Born-Infeld theory. In their generalization, they have found a one-parameter family of finite energy solutions in the case of the $S U(2)$ gauge group. In 2013, Hendi [13] proposed a nonlinear generalization of Maxwell electrodynamics which is called exponential electrodynamics $[14,15]$. The black hole solutions of Einstein's gravity in the presence of exponential electrodynamics in a 3+1-dimensional spacetime are obtained in [13]. In 2014, Gaete and Helayël-Neto introduced a new generalization of Maxwell electrodynamics which is known as logarithmic electrodynamics [14]. They proved that the classical selfenergy of a point charge in logarithmic electrodynamics is 
a finite value. Recently, a novel generalization of Born-Infeld electrodynamics is presented by Gaete and Helayël-Neto in which the authors show that the field energy of a pointlike charge is finite only for Born-Infeld like electrodynamics [15]. In [16], a nonlinear model for electrodynamics with manifestly broken gauge symmetry is proposed. In the above mentioned model, for nonlinear electrodynamics, there are nonsingular solitonic solutions which describe charged particles. Another interesting theory of nonlinear electrodynamics was proposed and developed by Heisenberg and his students Euler and Kockel [17-19]. They showed that classical electrodynamics must be corrected by nonlinear terms due to the vacuum polarization effects. In [20], the charged black hole solutions for Einstein-Euler-Heisenberg theory are obtained. There are three physical motivations in writing this paper. First, the exact solutions of nonlinear field equations are very important in theoretical physics. These solutions help us to obtain a better understanding of physical reality. According to the above statements, we attempt to obtain particular cylindrically symmetric solutions in Born-Infeld electrostatics. The search for spherically symmetric solutions in Born-Infeld electrostatics will be discussed in future works. Second, we want to show that the nonlinear corrections in electrodynamics are considerable only for very strong electric fields and extremely short spatial distances. Third, we hope to remove or at least modify the infinities which appear in Maxwell electrostatics. This paper is organized as follows. In Section 2, we study Lagrangian formulation of Abelian Born-Infeld model in the presence of an external source. The explicit forms of Gauss's law and the energy density of an electrostatic field for Born-Infeld electrostatics are obtained. In Section 3, we calculate the electric field together with the stored electrostatic energy per unit length for an infinite charged line and an infinitely long cylinder in Born-Infeld electrostatics. Summary and conclusions are presented in Section 4. Numerical estimations in Section 4 show that the nonlinear corrections to electric field of infinite charged line at large radial distances are negligible for weak electric fields. There are two appendices in this paper. In Appendix A, a generalized action functional for Abelian Born-Infeld model with an auxiliary scalar field in the presence of an external source is proposed. In Appendix B, we obtain the symmetric energy-momentum tensor for Abelian Born-Infeld model with an auxiliary scalar field. We use SI units throughout this paper. The metric of spacetime has the signature $(+,-,-,-)$.

\section{Lagrangian Formulation of Abelian Born- Infeld Model with an External Source}

The Lagrangian density for Abelian Born-Infeld model in a $3+1$-dimensional spacetime is [1-6]

$$
\mathscr{L}_{\mathrm{BI}}=\epsilon_{0} \beta^{2}\left\{1-\sqrt{1+\frac{c^{2}}{2 \beta^{2}} F_{\mu \nu} F^{\mu \nu}}\right\}-J^{\mu} A_{\mu},
$$

where $F_{\mu \nu}=\partial_{\mu} A_{\nu}-\partial_{\nu} A_{\mu}$ is the electromagnetic field tensor and $J^{\mu}=(c \rho, \mathbf{J})$ is an external source for the Abelian field $A^{\mu}=((1 / c) \phi, \mathbf{A})$. The parameter $\beta$ in (3) is called the nonlinearity parameter of the model. In the limit $\beta \rightarrow \infty$, (3) reduces to the Lagrangian density of the Maxwell field; that is,

$$
\left.\mathscr{L}_{\text {BI }}\right|_{\text {large } \beta}=\mathscr{L}_{M}+\mathcal{O}\left(\beta^{-2}\right),
$$

where $\mathscr{L}_{M}=-\left(1 / 4 \mu_{0}\right) F_{\mu \nu} F^{\mu \nu}-J^{\mu} A_{\mu}$ is the Maxwell Lagrangian density. The Euler-Lagrange equation for the Born-Infeld field $A^{\mu}$ is

$$
\frac{\partial \mathscr{L}_{\mathrm{BI}}}{\partial A_{\lambda}}-\partial_{\rho}\left(\frac{\partial \mathscr{L}_{\mathrm{BI}}}{\partial\left(\partial_{\rho} A_{\lambda}\right)}\right)=0 .
$$

If we substitute Lagrangian density (3) in the Euler-Lagrange equation (5), we will obtain the inhomogeneous Born-Infeld equations as follows:

$$
\partial_{\rho}\left(\frac{F^{\rho \lambda}}{\sqrt{1+\left(c^{2} / 2 \beta^{2}\right) F_{\mu \nu} F^{\mu \nu}}}\right)=\mu_{0} J^{\lambda} .
$$

The electromagnetic field tensor $F_{\mu \nu}$ satisfies the Bianchi identity:

$$
\partial_{\mu} F_{\nu \lambda}+\partial_{\nu} F_{\lambda \mu}+\partial_{\lambda} F_{\mu \nu}=0
$$

Equation (7) leads to the homogeneous Maxwell equations. In $3+1$-dimensional spacetime, the components of $F_{\mu \nu}$ can be written as follows:

$$
F_{\mu \nu}=\left(\begin{array}{cccc}
0 & \frac{E_{x}}{c} & \frac{E_{y}}{c} & \frac{E_{z}}{c} \\
-\frac{E_{x}}{c} & 0 & -B_{z} & B_{y} \\
-\frac{E_{y}}{c} & B_{z} & 0 & -B_{x} \\
-\frac{E_{z}}{c} & -B_{y} & B_{x} & 0
\end{array}\right) .
$$

Using (8), (6) and (7) can be written in the vector form as follows:

$$
\begin{aligned}
& \nabla \cdot\left(\frac{\mathbf{E}(\mathbf{x}, t)}{\sqrt{1-\left(\mathbf{E}^{2}(\mathbf{x}, t)-c^{2} \mathbf{B}^{2}(\mathbf{x}, t)\right) / \beta^{2}}}\right)=\frac{\rho(\mathbf{x}, t)}{\epsilon_{0}}, \\
& \nabla \times \mathbf{E}(\mathbf{x}, t)=-\frac{\partial \mathbf{B}(\mathbf{x}, t)}{\partial t}, \\
& \nabla \times\left(\frac{\mathbf{B}(\mathbf{x}, t)}{\sqrt{1-\left(\mathbf{E}^{2}(\mathbf{x}, t)-c^{2} \mathbf{B}^{2}(\mathbf{x}, t)\right) / \beta^{2}}}\right) \\
& =\mu_{0} \mathbf{J}(\mathbf{x}, t) \\
& +\frac{1}{c^{2}} \frac{\partial}{\partial t}\left(\frac{\mathbf{E}(\mathbf{x}, t)}{\sqrt{1-\left(\mathbf{E}^{2}(\mathbf{x}, t)-c^{2} \mathbf{B}^{2}(\mathbf{x}, t)\right) / \beta^{2}}}\right), \\
& \nabla \cdot \mathbf{B}(\mathbf{x}, t)=0 .
\end{aligned}
$$


The symmetric energy-momentum tensor for the Abelian Born-Infeld model in (3) has been obtained by Accioly [21] as follows:

$$
T_{\lambda}^{\mu}=\frac{1}{\mu_{0}}\left[\frac{F^{\mu \nu} F_{\nu \lambda}}{\Omega}+\frac{\beta^{2}}{c^{2}}(\Omega-1) \delta_{\lambda}^{\mu}\right],
$$

where $\Omega:=\sqrt{1+\left(c^{2} / 2 \beta^{2}\right) F_{\alpha \gamma} F^{\alpha \gamma}}$. The classical Born-Infeld equations (9) for an electrostatic field $\mathbf{E}(\mathbf{x})$ are

$$
\begin{gathered}
\nabla \cdot\left(\frac{\mathbf{E}(\mathbf{x})}{\sqrt{1-\mathbf{E}^{2}(\mathbf{x}) / \beta^{2}}}\right)=\frac{\rho(\mathbf{x})}{\epsilon_{0}}, \\
\nabla \times \mathbf{E}(\mathbf{x})=0 .
\end{gathered}
$$

Equations (11) and (12) are fundamental equations of BornInfeld electrostatics [2]. Using divergence theorem, the integral form of (11) can be written in the form

$$
\oint_{S} \frac{1}{\sqrt{1-\mathbf{E}^{2}(\mathbf{x}) / \beta^{2}}} \mathbf{E}(\mathbf{x}) \cdot \mathbf{n} d a=\frac{1}{\epsilon_{0}} \int_{V} \rho(\mathbf{x}) d^{3} x
$$

where $V$ is the three-dimensional volume enclosed by a twodimensional surface S. Equation (13) is Gauss's law in BornInfeld electrostatics. Using (8) and (10), the energy density of an electrostatic field in Born-Infeld theory is given by

$$
u(\mathbf{x})=\epsilon_{0} \beta^{2}\left(\frac{1}{\sqrt{1-\mathbf{E}^{2}(\mathbf{x}) / \beta^{2}}}-1\right)
$$

In the limit $\beta \rightarrow \infty$, the modified electrostatic energy density in (14) smoothly becomes the usual electrostatic energy density in Maxwell theory; that is,

$$
\left.u(\mathbf{x})\right|_{\text {large } \beta}=\frac{1}{2} \epsilon_{0} \mathbf{E}^{2}(\mathbf{x})+\mathcal{O}\left(\beta^{-2}\right) .
$$

\section{Calculation of Stored Electrostatic Energy per Unit Length for an Infinite Charged Line and an Infinitely Long Cylinder in Born-Infeld Electrostatics}

3.1. Infinite Charged Line. Let us consider an infinite charged line with a uniform positive linear charge density $\lambda$ which is located on the $z$-axis. Now, we find an expression for the electric field $\mathbf{E}(\mathbf{x})$ at a radial distance $\rho$ from the $z$-axis. Because of the cylindrical symmetry of the problem, the suitable Gaussian surface is a circular cylinder of radius $\rho$ and length $L$, coaxial with the $z$-axis (see Figure 1 ).

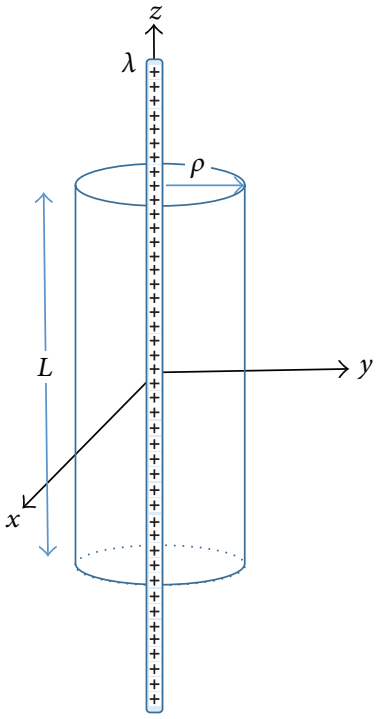

Figure 1: The Gaussian surface for an infinite charged line. The cylindrical symmetry of the problem implies that $\mathbf{E}(\mathbf{x})=E_{\rho}(\rho) \widehat{\mathbf{e}}_{\rho}$, where $\widehat{\mathbf{e}}_{\rho}$ is the radial unit vector in cylindrical coordinates $(\rho, \varphi, z)$.

Using the cylindrical symmetry of the problem together with the modified Gauss's law in (13), the electric field for the Gaussian surface in Figure 1 becomes

$$
\mathbf{E}(\mathbf{x})=\frac{\lambda}{2 \pi \epsilon_{0} \rho} \frac{1}{\sqrt{1+\left(\lambda / 2 \pi \epsilon_{0} \beta \rho\right)^{2}}} \widehat{\mathbf{e}}_{\rho} .
$$

In contrast with Maxwell electrostatics, the electric field $\mathbf{E}(\mathbf{x})$ in (16) has a finite value on the $z$-axis; that is,

$$
\lim _{\rho \rightarrow 0} \mathbf{E}(\mathbf{x})=\beta \widehat{\mathbf{e}}_{\rho}
$$

At large radial distances from the $z$-axis, the asymptotic behavior of the electric field in (16) is given by

$$
\mathbf{E}(\mathbf{x})=\frac{\lambda}{2 \pi \epsilon_{0} \rho} \widehat{\mathbf{e}}_{\rho}-\frac{\lambda^{3}}{16 \pi^{3} \epsilon_{0}^{3} \beta^{2} \rho^{3}} \widehat{\mathbf{e}}_{\rho}+\mathcal{O}\left(\rho^{-5}\right) .
$$

The first term on the right-hand side of (18) shows the electric field of an infinite charged line in Maxwell electrostatics, while the second and higher order terms in (18) show the effect of nonlinear corrections. By putting (16) in (14), the electrostatic energy density for an infinite charged line in Born-Infeld electrostatics can be written as follows:

$$
u(\mathbf{x})=\epsilon_{0} \beta^{2}\left(\sqrt{1+\left(\frac{\lambda}{2 \pi \epsilon_{0} \beta \rho}\right)^{2}}-1\right) .
$$




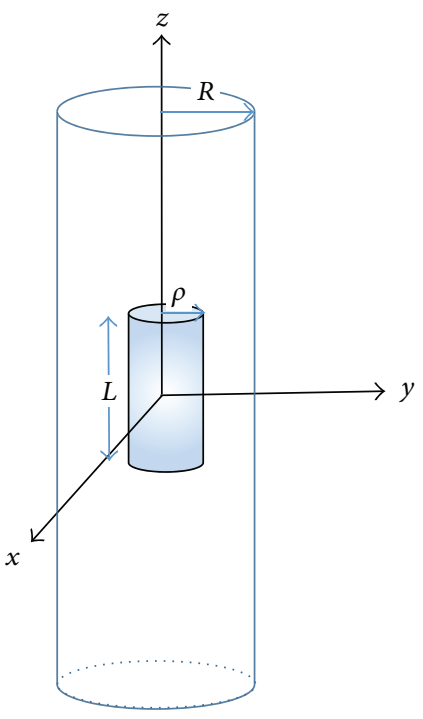

(a)

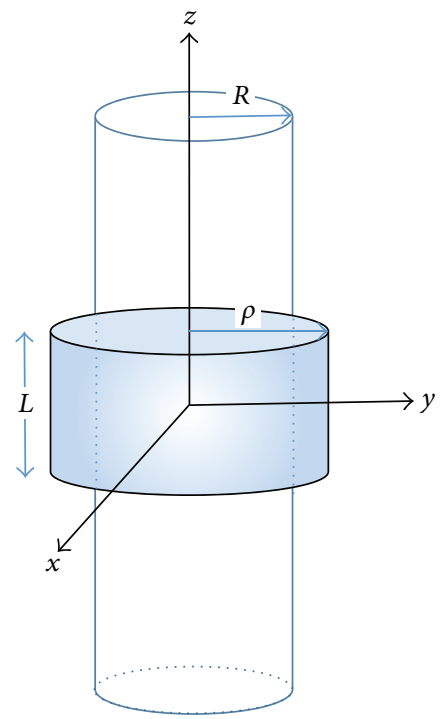

(b)

Figure 2: The Gaussian surface for an infinitely long cylinder of radius $R$. (a) Inside the cylinder $(\rho<R)$. (b) Outside the cylinder $(\rho>R)$.

Using (19), the stored electrostatic energy per unit length for an infinite charged line in the radial interval $0 \leq \rho \leq \Lambda$ is given by

$$
\begin{aligned}
& \frac{U}{L}=\int_{0}^{\Lambda} \int_{0}^{2 \pi} u(\mathbf{x}) \rho d \rho d \varphi \\
&=2 \pi \epsilon_{0} \beta^{2}\left\{\frac{\Lambda}{2} \sqrt{\Lambda^{2}+\left(\frac{\lambda}{2 \pi \epsilon_{0} \beta}\right)^{2}}-\frac{\Lambda^{2}}{2}+\frac{1}{2}\left(\frac{\lambda}{2 \pi \epsilon_{0} \beta}\right)^{2}\right. \\
&\left.\cdot \ln \left(\frac{\Lambda+\sqrt{\Lambda^{2}+\left(\lambda / 2 \pi \epsilon_{0} \beta\right)^{2}}}{\left(\lambda / 2 \pi \epsilon_{0} \beta\right)}\right)\right\} .
\end{aligned}
$$

It is necessary to note that the above value for $U / L$ has an infinite value in Maxwell theory. In the limit of large $\beta$, the expression for $U / L$ in (20) diverges logarithmically as $\ln \beta$. Hence, it seems that the finite regularization parameter $\beta$ removes the logarithmic divergence in (20).

3.2. Infinitely Long Cylinder. In this subsection, we determine the electric field $\mathbf{E}(\mathbf{x})$ and stored electrostatic energy per unit length for an infinitely long cylinder of radius $R$ and uniform positive volume charge density $\tau$. As in the previous subsection, we assume that the Gaussian surface is a cylindrical closed surface of radius $\rho$ and length $L$ with a common axis with the infinitely long cylinder (see Figure 2).

According to modified Gauss's law in (13), the electric field for the Gaussian surfaces in Figure 2 is given by

$$
\mathbf{E}(\mathbf{x})= \begin{cases}\frac{\tau \rho}{2 \epsilon_{0}} \frac{1}{\sqrt{1+\left(\tau \rho / 2 \epsilon_{0} \beta\right)^{2}}} \widehat{\mathbf{e}}_{\rho} ; & \rho<R, \\ \frac{\tau R^{2}}{2 \epsilon_{0} \rho} \frac{1}{\sqrt{1+\left(\tau R^{2} / 2 \epsilon_{0} \beta \rho\right)^{2}}} \widehat{\mathbf{e}}_{\rho} ; & \rho>R .\end{cases}
$$

For the large values of the nonlinearity parameter $\beta$, the behavior of the electric field $\mathbf{E}(\mathbf{x})$ in (21) is as follows:

$$
\mathbf{E}(\mathbf{x})= \begin{cases}\frac{\tau \rho}{2 \epsilon_{0}} \widehat{\mathbf{e}}_{\rho}-\frac{\tau^{3} \rho^{3}}{16 \epsilon_{0}^{3} \beta^{2}} \widehat{\mathbf{e}}_{\rho}+\mathcal{O}\left(\beta^{-4}\right) ; & \rho<R, \\ \frac{\tau R^{2}}{2 \epsilon_{0} \rho} \widehat{\mathbf{e}}_{\rho}-\frac{\tau^{3} R^{6}}{16 \epsilon_{0}^{3} \beta^{2} \rho^{3}} \widehat{\mathbf{e}}_{\rho}+\mathcal{O}\left(\beta^{-4}\right) ; & \rho>R .\end{cases}
$$

Hence, for the large values of $\beta$, the electric field $\mathbf{E}(\mathbf{x})$ in (22) becomes the electric field of an infinitely long cylinder in Maxwell electrostatics. If we substitute (21) into (14), we will obtain the electrostatic energy density for an infinitely long cylinder in Born-Infeld electrostatics as follows:

$$
u(\mathbf{x})= \begin{cases}\epsilon_{0} \beta^{2}\left(\sqrt{1+\left(\frac{\tau \rho}{2 \epsilon_{0} \beta}\right)^{2}}-1\right) ; & \rho<R, \\ \epsilon_{0} \beta^{2}\left(\sqrt{1+\left(\frac{\tau R^{2}}{2 \epsilon_{0} \beta \rho}\right)^{2}}-1\right) ; & \rho>R .\end{cases}
$$

Using (23), the stored electrostatic energy per unit length for an infinitely long cylinder in the radial interval $0 \leq \rho \leq$ $\Lambda(\Lambda>R)$ is given by

$$
\begin{aligned}
\frac{U}{L}=2 \pi \epsilon_{0} \beta^{2}\{ & \int_{0}^{R}\left(\sqrt{1+\left(\frac{\tau \rho}{2 \epsilon_{0} \beta}\right)^{2}}-1\right) \rho d \rho \\
& \left.+\int_{R}^{\Lambda}\left(\sqrt{1+\left(\frac{\tau R^{2}}{2 \epsilon_{0} \beta \rho}\right)^{2}}-1\right) \rho d \rho\right\}
\end{aligned}
$$




$$
\begin{aligned}
=2 \pi \epsilon_{0} \beta^{2}\{ & -\frac{\Lambda^{2}}{2}+\left(\frac{2 \epsilon_{0} \beta}{\tau \sqrt{3}}\right)^{2}\left[\left(1+\left(\frac{\tau R}{2 \epsilon_{0} \beta}\right)^{2}\right)^{3 / 2}-1\right] \\
& +\frac{\Lambda^{2}}{2} \sqrt{1+\left(\frac{\tau R^{2}}{2 \epsilon_{0} \beta \Lambda}\right)^{2}} \\
& -\frac{R^{2}}{2} \sqrt{1+\left(\frac{\tau R}{2 \epsilon_{0} \beta}\right)^{2}}+\frac{1}{2}\left(\frac{\tau R^{2}}{2 \epsilon_{0} \beta}\right)^{2} \\
& \left.\cdot \ln \left(\frac{\Lambda+\Lambda \sqrt{1+\left(\tau R^{2} / 2 \epsilon_{0} \beta \Lambda\right)^{2}}}{R+R \sqrt{1+\left(\tau R / 2 \epsilon_{0} \beta\right)^{2}}}\right)\right\}
\end{aligned}
$$

In the limit of large $\beta$, the expression for $U / L$ in (24) can be expanded in powers of $1 / \beta^{2}$ as follows:

$$
\left.\frac{U}{L}\right|_{\text {large } \beta}=\frac{\pi \tau^{2} R^{4}}{4 \epsilon_{0}}\left(\frac{1}{4}+\ln \frac{\Lambda}{R}\right)+\mathcal{O}\left(\beta^{-2}\right) .
$$

The first term on the right-hand side of (25) shows the stored electrostatic energy per unit length for an infinitely long cylinder in the radial interval $0 \leq \rho \leq \Lambda(\Lambda>R)$ in Maxwell electrostatics.

\section{Summary and Conclusions}

In 1934, Born and Infeld introduced a nonlinear generalization of Maxwell electrodynamics, in which the classical selfenergy of a point charge like electron became a finite value [1]. We showed that, in the limit of large $\beta$, the modified Gauss's law in Born-Infeld electrostatics is

$$
\begin{aligned}
\oint_{S}[1 & \left.+\frac{1}{2} \frac{\mathbf{E}^{2}(\mathbf{x})}{\beta^{2}}+\frac{3}{8} \frac{\left(\mathbf{E}^{2}(\mathbf{x})\right)^{2}}{\beta^{4}}+\mathcal{O}\left(\beta^{-6}\right)\right] \mathbf{E}(\mathbf{x}) \cdot \mathbf{n} d a \\
& =\frac{1}{\epsilon_{0}} \int_{V} \rho(\mathbf{x}) d^{3} x .
\end{aligned}
$$

By using the modified Gauss's law in (13), we calculated the electric field of an infinite charged line and an infinitely long cylinder in Born-Infeld electrostatics. The stored electrostatic energy per unit length for the above configurations of charge density has been calculated in the framework of Born-Infeld electrostatics. Born and Infeld attempted to determine $\beta$ by equating the classical self-energy of the electron in their theory with its rest mass energy. They obtained the following numerical value for the nonlinearity parameter $\beta[1]$ :

$$
\beta=1.2 \times 10^{20} \mathrm{~V} / \mathrm{m} .
$$

In 1973, Soff et al. [22] have estimated a lower bound on $\beta$. This lower bound on $\beta$ is

$$
\beta \geq 1.7 \times 10^{22} \mathrm{~V} / \mathrm{m}
$$

Recent studies on photonic processes in Born-Infeld theory show that the numerical value of $\beta$ is close to $1.2 \times 10^{20} \mathrm{~V} / \mathrm{m}$ in (27) [23]. In order to obtain a better understanding of nonlinear effects in Born-Infeld electrostatics, let us estimate the numerical value of the second term on the right-hand side of (18). For this purpose, we rewrite (18) as follows:

$$
\mathbf{E}(\mathbf{x})=\mathbf{E}_{0}(\mathbf{x})+\Delta \mathbf{E}(\mathbf{x})+\mathcal{O}\left(\rho^{-5}\right)
$$

where

$$
\begin{gathered}
\mathbf{E}_{0}(\mathbf{x}):=\frac{\lambda}{2 \pi \epsilon_{0} \rho} \widehat{\mathbf{e}}_{\rho}, \\
\Delta \mathbf{E}(\mathbf{x}):=-\frac{\lambda^{3}}{16 \pi^{3} \epsilon_{0}^{3} \beta^{2} \rho^{3}} \widehat{\mathbf{e}}_{\rho} .
\end{gathered}
$$

Using (30), the ratio of $\Delta \mathbf{E}(\mathbf{x})$ to $\mathbf{E}_{0}(\mathbf{x})$ is given by

$$
\frac{|\Delta \mathbf{E}(\mathbf{x})|}{\left|\mathbf{E}_{0}(\mathbf{x})\right|}=\frac{1}{2} \frac{\mathbf{E}_{0}^{2}(\mathbf{x})}{\beta^{2}}
$$

Let us assume the following approximate but realistic values [24]:

$$
\begin{gathered}
L=1.80 \mathrm{~m}, \quad \rho=0.10 \mathrm{~m}, \quad Q=+24 \mu C, \\
\lambda=1.33 \times 10^{-5} \mathrm{C} / \mathrm{m} .
\end{gathered}
$$

By putting (27) and (32) into (31), we get

$$
|\Delta \mathbf{E}(\mathbf{x})| \approx 2 \times 10^{-28}\left|\mathbf{E}_{0}(\mathbf{x})\right|
$$

Finally, if we put (28) and (32) in (31), we obtain

$$
|\Delta \mathbf{E}(\mathbf{x})| \lesssim 10^{-32}\left|\mathbf{E}_{0}(\mathbf{x})\right|
$$

In fact, as is clear from (33) and (34), the nonlinear corrections to electric field in (18) are very small for weak electric fields. The authors of [25] have suggested a nonlinear generalization of Maxwell electrodynamics. In their generalization, the electric field of a point charge is singular at the position of the point charge but the classical selfenergy of the point charge has a finite value. Recently, Kruglov $[26,27]$ has proposed two different models for nonlinear electrodynamics. In these models, both the electric field of a point charge at the position of the point charge and the classical self-energy of the point charge have finite values. In future works, we hope to study the problems discussed in this research from the viewpoint of [25-27]. 


\section{Appendices}

\section{A. A Generalized Action Functional for Abelian Born-Infeld Model with an Auxiliary Scalar Field}

Let us consider the following action functional:

$$
\begin{gathered}
S(A, \psi) \\
=\frac{1}{c} \int_{t_{0}}^{t} \int_{\mathbb{R}^{3}}\left[\epsilon _ { 0 } \beta ^ { 2 } \left(1-\omega_{1} \psi^{\lambda_{1}}\left(1+\frac{c^{2}}{2 \beta^{2}} F_{\mu \nu} F^{\mu \nu}\right)\right.\right. \\
\left.\left.-\omega_{2} \psi^{\lambda_{2}}\right)-J^{\mu} A_{\mu}\right] d^{4} x
\end{gathered}
$$

where $\psi$ is an auxiliary scalar field and $\omega_{1}, \lambda_{1}, \omega_{2}$, and $\lambda_{2}$ are four nonzero constants. The variation of (A.1) with respect to $\psi$ and $A_{\mu}$ leads to the following classical field equations:

$$
\begin{gathered}
\psi=\left[-\frac{\omega_{2} \lambda_{2}}{\omega_{1} \lambda_{1}}\left(1+\frac{c^{2}}{2 \beta^{2}} F_{\mu \nu} F^{\mu \nu}\right)^{-1}\right]^{1 /\left(\lambda_{1}-\lambda_{2}\right)}, \\
\partial_{\mu}\left(\psi^{\lambda_{1}} F^{\mu \nu}\right)=\frac{\mu_{0}}{2 \omega_{1}} J^{\nu} .
\end{gathered}
$$

Substituting (A.2) into (A.3), we obtain the following classical field equation:

$$
\begin{aligned}
\partial_{\mu}\left\{\left[-\frac{\omega_{2} \lambda_{2}}{\omega_{1} \lambda_{1}}\left(1+\frac{c^{2}}{2 \beta^{2}} F_{\alpha \gamma} F^{\alpha \gamma}\right)^{-1}\right]^{\lambda_{1} /\left(\lambda_{1}-\lambda_{2}\right)} F^{\mu \nu}\right\} \\
=\frac{\mu_{0}}{2 \omega_{1}} J^{\nu} .
\end{aligned}
$$

By choosing $\lambda_{1}=\lambda, \lambda_{2}=-\lambda$ and $\omega_{1}=\omega, \omega_{2}=(1 / 4 \omega)(\omega>$ 0 ), (A.1) and (A.4) can be written as follows:

$$
\begin{array}{r}
S(A, \psi) \\
=\frac{1}{c} \int_{t_{0}}^{t} \int_{\mathbb{R}^{3}}\left[\epsilon _ { 0 } \beta ^ { 2 } \left(1-\omega \psi^{\lambda}\left(1+\frac{c^{2}}{2 \beta^{2}} F_{\mu \nu} F^{\mu \nu}\right)\right.\right. \\
\left.\left.-\frac{1}{4 \omega} \psi^{-\lambda}\right)-J^{\mu} A_{\mu}\right] d^{4} x, \\
\partial_{\mu}\left(\frac{F^{\mu \nu}}{\sqrt{1+\left(c^{2} / 2 \beta^{2}\right) F_{\alpha \gamma} F^{\alpha \gamma}}}\right)=\mu_{0} J^{\nu} .
\end{array}
$$

Equation (A.5) is the generalized action functional for Abelian Born-Infeld model with an auxiliary scalar field $\psi$. Also, (A.6) is the inhomogeneous Born-Infeld equation (see (6)). If we choose $\omega=1 / 2$ and $\lambda=1$ in (A.5), we will obtain the following action functional:

$$
\begin{array}{r}
S(A, \psi) \\
=\frac{1}{c} \int_{t_{0}}^{t} \int_{\mathbb{R}^{3}}\left[\epsilon _ { 0 } \beta ^ { 2 } \left(1-\frac{\psi}{2}\left(1+\frac{c^{2}}{2 \beta^{2}} F_{\mu \nu} F^{\mu \nu}\right)\right.\right. \\
\left.\left.-\frac{1}{2 \psi}\right)-J^{\mu} A_{\mu}\right] d^{4} x .
\end{array}
$$

The above action functional was presented by Tseytlin in his studies on low energy dynamics of $D$-branes [28].

\section{B. The Symmetric Energy-Momentum Tensor for Abelian Born-Infeld Model with an Auxiliary Scalar Field}

In this appendix, we want to obtain the symmetric energymomentum tensor for Abelian Born-Infeld model with an auxiliary scalar field. According to (A.5), the Lagrangian density for Abelian Born-Infeld model with an auxiliary scalar field $\psi$ in the absence of external source $J^{\mu}$ is

$$
\mathscr{L}=\epsilon_{0} \beta^{2}\left(1-\omega \psi^{\lambda}\left(1+\frac{c^{2}}{2 \beta^{2}} F_{\mu \nu} F^{\mu \nu}\right)-\frac{1}{4 \omega} \psi^{-\lambda}\right) .
$$

From (B.1), we obtain the following classical field equations:

$$
\begin{gathered}
\psi^{\lambda}=\frac{1}{2 \omega}\left(1+\frac{c^{2}}{2 \beta^{2}} F_{\mu \nu} F^{\mu \nu}\right)^{-1 / 2}, \\
\partial_{\mu}\left(\psi^{\lambda} F^{\mu \nu}\right)=0 .
\end{gathered}
$$

The canonical energy-momentum tensor for (B.1) is

$$
\begin{aligned}
T_{\gamma}^{\alpha} & \frac{\partial \mathscr{L}}{\partial\left(\partial_{\alpha} A_{\eta}\right)}\left(\partial_{\gamma} A_{\eta}\right)+\frac{\partial \mathscr{L}}{\partial\left(\partial_{\alpha} \psi\right)}\left(\partial_{\gamma} \psi\right)-\delta_{\gamma}^{\alpha} \mathscr{L} \\
= & -2 \epsilon_{0} c^{2} \omega \psi^{\lambda} F^{\alpha \eta}\left(\partial_{\gamma} A_{\eta}\right) \\
& -\epsilon_{0} \beta^{2} \delta_{\gamma}^{\alpha}\left(1-\omega \psi^{\lambda}\left(1+\frac{c^{2}}{2 \beta^{2}} F_{\mu \nu} F^{\mu \nu}\right)-\frac{1}{4 \omega} \psi^{-\lambda}\right) .
\end{aligned}
$$

Using (B.2), the canonical energy-momentum tensor $T_{\gamma}^{\alpha}$ in (B.3) can be rewritten as follows:

$$
\begin{aligned}
& T_{\gamma}^{\alpha} \\
& =-\epsilon_{0} c^{2}\left(1+\frac{c^{2}}{2 \beta^{2}} F_{\mu \nu} F^{\mu \nu}\right)^{-1 / 2} F^{\alpha \eta} F_{\gamma \eta} \\
& \quad-\epsilon_{0} \beta^{2} \delta_{\gamma}^{\alpha}\left(1-\left(1+\frac{c^{2}}{2 \beta^{2}} F_{\mu \nu} F^{\mu \nu}\right)^{1 / 2}\right)+\partial_{\eta} M_{\gamma}^{\eta \alpha},
\end{aligned}
$$


where

$$
\begin{gathered}
M_{\gamma}^{\eta \alpha}:=\frac{1}{\mu_{0}} \frac{F^{\eta \alpha}}{\sqrt{1+\left(c^{2} / 2 \beta^{2}\right) F_{\mu \nu} F^{\mu \nu}}} A_{\gamma}, \\
M_{\gamma}^{\alpha \eta}=-M_{\gamma}^{\eta \alpha} .
\end{gathered}
$$

After dropping the total divergence term $\partial_{\eta} M_{\gamma}^{\eta \alpha}$ in (B.4), we get the following expression for the symmetric energymomentum tensor:

$$
\begin{aligned}
T_{\gamma}^{\alpha}= & -\epsilon_{0} c^{2}\left(1+\frac{c^{2}}{2 \beta^{2}} F_{\mu \nu} F^{\mu \nu}\right)^{-1 / 2} F^{\alpha \eta} F_{\gamma \eta} \\
& -\epsilon_{0} \beta^{2} \delta_{\gamma}^{\alpha}\left(1-\left(1+\frac{c^{2}}{2 \beta^{2}} F_{\mu \nu} F^{\mu \nu}\right)^{1 / 2}\right) .
\end{aligned}
$$

If we use (8) and (B.6), we will obtain the electrostatic energy density for Abelian Born-Infeld model with an auxiliary scalar field as follows:

$$
u(\mathbf{x})=T_{0}^{0}(\mathbf{x})=\epsilon_{0} \beta^{2}\left(\frac{1}{\sqrt{1-\mathbf{E}^{2}(\mathbf{x}) / \beta^{2}}}-1\right) .
$$

\section{Conflict of Interests}

The authors declare that there is no conflict of interests regarding the publication of this paper.

\section{Acknowledgment}

The authors would like to thank the referees for their careful reading and constructive comments.

\section{References}

[1] M. Born and L. Infeld, "Foundations of the new field theory," Proceedings of the Royal Society of London A, vol. 144, p. 425, 1934.

[2] D. Fortunato, L. Orsina, and L. Pisani, "Born-Infeld type equations for electrostatic fields," Journal of Mathematical Physics, vol. 43, no. 11, pp. 5698-5706, 2002.

[3] S. H. Mazharimousavi and M. Halilsoy, "Ground state H-atom in born-infeld teory," Foundations of Physics, vol. 42, no. 4, pp. 524-530, 2012.

[4] L. P. De Assis, P. Gaete, J. A. Helayel-Neto, and S. O. Vellozo, "Aspects of magnetic field configurations in planar nonlinear electrodynamics," International Journal of Theoretical Physics, vol. 51, no. 2, pp. 477-486, 2012.

[5] Z. K. Vad, "Some solutions of Einstein-Born-Infeld field equations," Acta Physica Hungarica, vol. 63, no. 3-4, pp. 353-364, 1988.

[6] P. Gaete and I. Schmidt, "Coulomb's law modification in nonlinear and in noncommutative electrodynamics," International Journal of Modern Physics A, vol. 19, no. 20, pp. 3427-3437, 2004.

[7] C. G. Callan and J. M. Maldacena, "Brane dynamics from the Born-Infeld action," Nuclear Physics B, vol. 513, no. 1-2, pp. 198212, 1998.
[8] G. W. Gibbons, "Born-Infeld particles and Dirichlet $p$-branes," Nuclear Physics. B, vol. 514, no. 3, pp. 603-639, 1998.

[9] G. W. Gibbons, "Aspects of born-infeld theory and string/Mtheory," Revista Mexicana de Física, vol. 49, supplement 1, pp. 19-29, 2003.

[10] B. Zwiebach, A First Course in String Theory, Cambridge University Press, Cambridge, UK, 2nd edition, 2009.

[11] H. Q. Lu, "Phantom cosmology with a nonlinear born-infeld type scalar field," International Journal of Modern Physics D, vol. 14, no. 2, p. 355, 2005.

[12] E. Serie, T. Masson, and R. Kerner, "Non-abelian generalization of Born-Infeld theory inspired by noncommutative geometry," Physical Review D, vol. 68, no. 12, Article ID 125003, 2003.

[13] S. H. Hendi, "Asymptotic Reissner-Nordström black holes," Annals of Physics, vol. 333, pp. 282-289, 2013.

[14] P. Gaete and J. Helayël-Neto, "Finite field-energy and interparticle potential in logarithmic electrodynamics," The European Physical Journal C, vol. 74, article 2816, 2014.

[15] P. Gaete and J. Helayël-Neto, "Remarks on nonlinear electrodynamics," The European Physical Journal C, vol. 74, no. 11, article 3182, 2014.

[16] N. Riazi and M. Mohammadi, "Composite lane-emden equation as a nonlinear poisson equation," International Journal of Theoretical Physics, vol. 51, no. 4, pp. 1276-1283, 2012.

[17] H. Euler and B. Kockel, "Über die streuung von licht an licht nach der diracschen theorie," Naturwissenschaften, vol. 23, no. 15, pp. 246-247, 1935.

[18] H. Euler, "Ober die Streuung von Licht an Licht nach der Diracschen Theorie," Annalen der Physik (Leipzig), vol. 26, pp. 398-448, 1936.

[19] W. Heisenberg and H. Euler, "Folgerungen aus der diracschen theorie des positrons," Zeitschrift für Physik, vol. 98, no. 11-12, pp. 714-732, 1936.

[20] R. Ruffini, Y.-B. Wu, and S.-S. Xue, "Einstein-Euler-Heisenberg theory and charged black holes," Physical Review D, vol. 88, Article ID 085004, 2013.

[21] A. Accioly, "Energy and momentum for the electromagnetic field described by three outstanding electrodynamics," American Journal of Physics, vol. 65, no. 9, pp. 882-887, 1997.

[22] G. Soff, J. Rafelski, and W. Greiner, "Lower bound to limiting fields in nonlinear electrodynamics," Physical Review A, vol. 7, no. 3, pp. 903-907, 1973.

[23] J. M. Davila, C. Schubert, and M. A. Trejo, "Photonic processes in Born-Infeld theory," International Journal of Modern Physics A. Particles and Fields. Gravitation. Cosmology, vol. 29, Article ID 1450174, 2014.

[24] D. Halliday, R. Resnick, and J. Walker, Fundamentals of Physics Extended, Wiley, 10th edition, 2014.

[25] C. V. Costa, D. M. Gitman, and A. E. Shabad, "Finite fieldenergy of a point charge in QED," http://arxiv.org/abs/1312 .0447 .

[26] S. I. Kruglov, "A model of nonlinear electrodynamics," Annals of Physics, vol. 353, pp. 299-306, 2015.

[27] S. I. Kruglov, "Nonlinear arcsin-electrodynamics," http://arxiv .org/abs/1410.7633.

[28] A. A. Tseytlin, "Self-duality of Born-Infeld action and Dirichlet 3-brane of type IIB superstring theory," Nuclear Physics B, vol. 469, no. 1-2, pp. 51-67, 1996. 

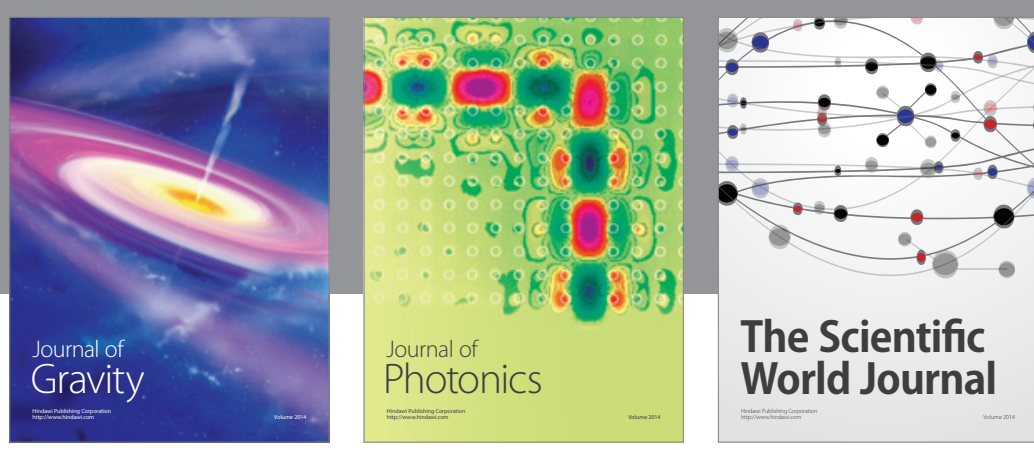

The Scientific World Journal
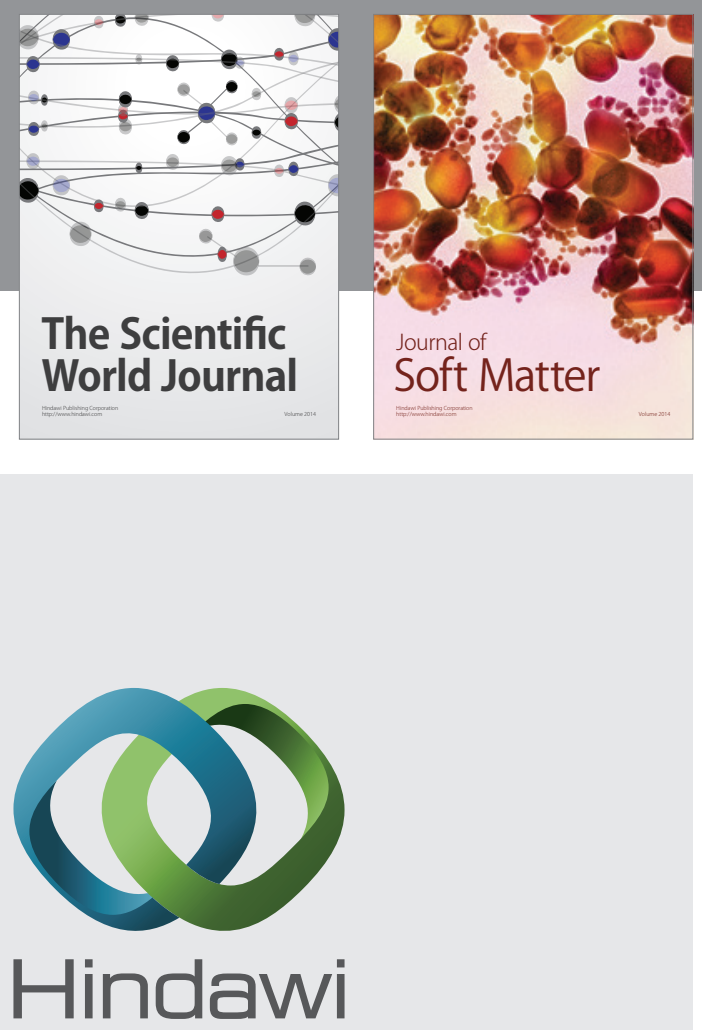

Submit your manuscripts at

http://www.hindawi.com

nternational Journal of

Statistical Mechanics
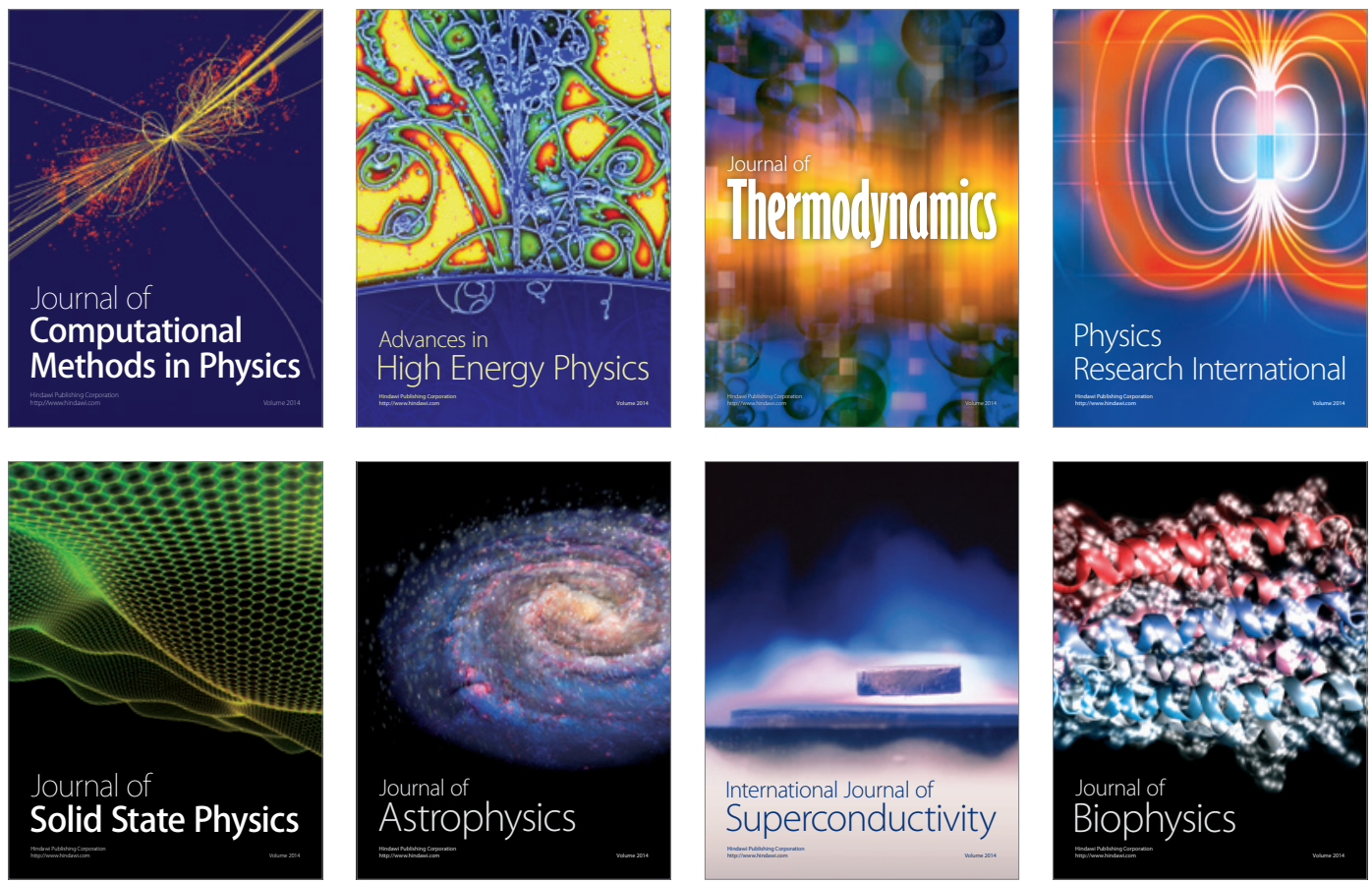
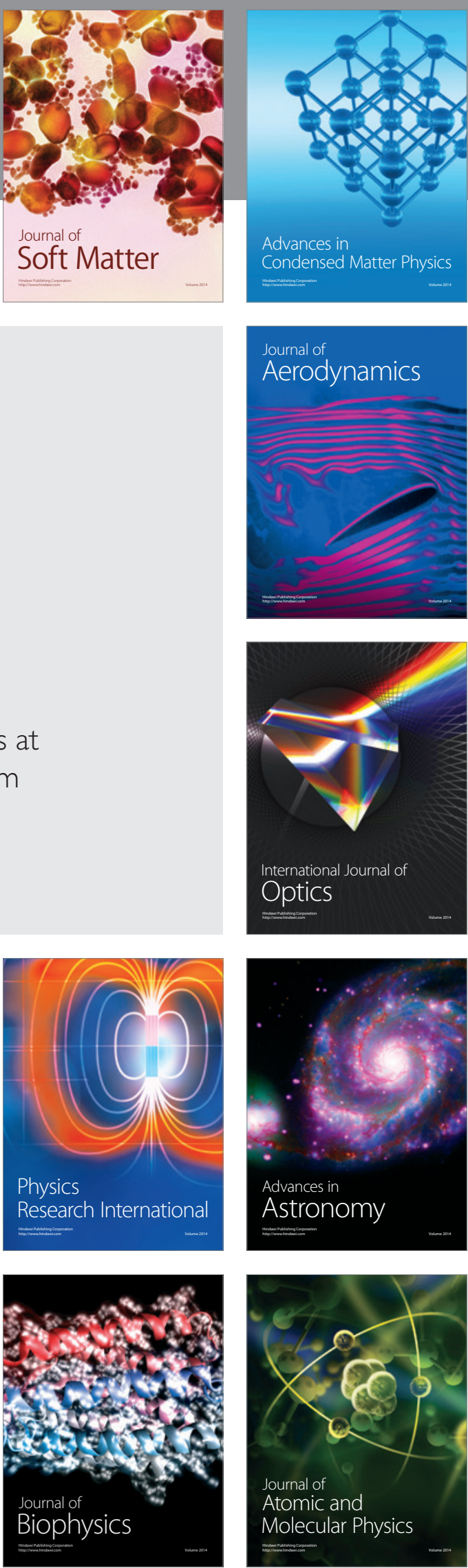